\title{
Association of Renal Insufficiency With In-Hospital Mortality Among Japanese Patients With Acute Myocardial Infarction Undergoing Percutaneous Coronary Interventions
}

\author{
Yoshihisa HiRAKAwA, ${ }^{1}$ MD, Yuichiro MASUdA, ${ }^{1} \mathrm{MD}$, \\ Masafumi KuzuYA, ${ }^{1}$ MD, Akihisa IGUCHI, ${ }^{1} \mathrm{MD}$, Takaya KimAtA, ${ }^{1} \mathrm{MD}$, \\ and Kazumasa UEMURA, ${ }^{2} \mathrm{MD}$
}

\section{SUMMARY}

It is not yet clear whether a difference in in-hospital morality between patients with and without renal insufficiency undergoing percutaneous coronary intervention (PCI) exists. Therefore, the aim of the present study was to investigate if such as association exists in Japan. Data from the Tokai Acute Myocardial Infarction Study II were used. This was a prospective study of all 3274 patients admitted with acute myocardial infarction (AMI) to the 15 participating hospitals from 2001 to 2003. We abstracted the baseline and procedural characteristics as well as in-hospital mortality from detailed chart reviews. Patients were stratified into 2 groups according to the estimated creatinine clearance on admission. The creatinine clearance values were available in 2116,107 of whom had renal insufficiency. The patients with renal insufficiency were more likely to be older, female, not independent in their daily activities, have lower body mass index and higher heart rate values on admission, lower prevalences of hypercholesterolemia and peptic ulcers, greater prevalences of diabetes, angina, previous heart failure, previous renal failure, previous cerebrovascular disease, aortic aneurysm, worse clinical course such as bleeding, and a multivessel coronary disease. Vasopressors, an intra-aortic balloon pump, and mechanical ventilation were frequently used in the patients with renal insufficiency, while thrombolytics were used less frequently. The patients with renal insufficiency had a higher inhospital mortality rate than those without. Multivariate analysis identified renal insufficiency as an independent predictor of in-hospital death. The results suggest that renal insufficiency is an independent predictor of in-hospital death among AMI patients undergoing PCI. (Int Heart J 2006; 47: 745-752)

Key words: Renal failure, In-hospital mortality, Percutaneous coronary intervention, Acute myocardial infarction, Japan

From the ${ }^{1}$ Department of Geriatrics, Nagoya University Graduate School of Medicine, ${ }^{2}$ Center of Medical Education, Nagoya University School of Medicine, Aichi, Japan.

Address for correspondence: Yoshihisa Hirakawa, MD, Department of Geriatrics, Nagoya University Graduate School of

Medicine, 65 Tsuruma-cho, Showa-ku, Nagoya, Aichi 466-8550, Japan.

Received for publication May 10, 2006.

Revised and accepted September 7, 2006. 
IT is well known that hypertension and diabetes mellitus are strongly related to chronic renal failure and the growth of elderly hypertensive and/or diabetic patients has triggered an increase in the number of hypertensive and/or diabetic patients with renal insufficiency. ${ }^{1-3)}$ Although previous studies have indicated that the clinical outcome of patients with chronic kidney disease is poor ${ }^{4-11)}$ in Western countries, the association has not yet been well established in patients undergoing percutaneous coronary intervention (PCI) and thus remains unclear, especially in non-Western countries such as Japan. Therefore, we investigated the association of renal insufficiency with in-hospital mortality among Japanese patients with AMI undergoing PCIs.

\section{MethodS}

Study population: The Tokai Acute Myocardial Infarction Study II (TAMISII) ${ }^{12)}$ is a multihospital prospective observational study conducted in the Tokai region of Japan. General patient recruitment for TAMIS-II began in January 2001 and continued through December 2003. The 3274 study subjects were adult patients who were hospitalized for newly diagnosed AMI at 15 different acute care hospitals. Their diagnoses were confirmed by a later chart review. Regarding the enrollment of participant hospitals, we selected major hospitals which had a staff exchange program with Nagoya University Hospital where we are based. These hospitals were sent a prospectus of our research project. A total of 15 hospitals, which were municipal or nonprofit general hospitals equipped to perform PCI, agreed to take part in the study.

Data collection: We abstracted the baseline and procedural characteristics and inhospital outcomes from detailed chart reviews which included both physician notes and nurse notes by physicians or nurses skilled at collecting medical records. The questionnaire contained information on age, sex, activities of daily living (ADL), medical history, and medical condition including body temperature, heart rate, systolic blood pressure, body mass index, bleeding, pulmonary edema, Killip score at hospital admission, locations of the MIs, max CPK, ejection fraction, number of coronary arteries narrowed $>75 \%$ according to the AHA classification including the left main coronary artery, and procedural characteristics during hospitalization (transfer to ICU/CCU, thrombolytics, vasopressor, IABP, mechanical ventilation, emergency PCI, and stent placement). Pulmonary edema was confirmed by an x-ray examination. In addition, the site of the MIs and the ejection fraction were confirmed by an ultrasound-echocardiogram examination. A history of comorbid conditions was recorded as present if documented in the medical charts.

Assessment of renal function: Renal function was assessed using the Cockroft- 
Gault formula: creatinine clearance $(\mathrm{CC})(\mathrm{mL} / \mathrm{min})=((140$-age $) \times$ body weight $(\mathrm{kg})) / 72 \times$ serum creatinine $(\mathrm{mg} / \mathrm{dL}))(\times 0.85$ for women $) .{ }^{13)}$ The serum creatinine value and the information were obtained in the emergency department or at the time of hospital admission at each participating TAMIS-II hospital. Because we did not identify patients who were on dialysis, patients on dialysis were analyzed together with patients who were not. Patients were divided into 2 groups according to their level of $\mathrm{CC}$ (insufficient renal function: $\mathrm{CC}<30$, normal renal function: $\mathrm{CC} \geqq 30$ )

Statistical analysis: We compared the baseline and procedural characteristics and the clinical outcomes between the patients with and those without renal insufficiency undergoing PCI. We did not include patients whose creatinine values were missing from our analysis. Statistical analysis was performed using the chisquare test for categorical variables and the unpaired $t$-test for continuous variables. We also performed a multivariable logistic regression analysis to assess the independence of the association between renal insufficiency and in-hospital death, after adjusting for the baseline characteristics, location of the MIs, max CPK, ejection fraction, and number of coronary arteries narrowed $>75 \%$ in the AHA classification that differed significantly between the 2 groups. Univariate predictors of those outcomes with a $P$ value of less than 0.05 were allowed to enter the model. We present the results as odds ratios and $95 \%$ confidence intervals.

\section{Results}

A baseline creatinine clearance on admission was available in $2116(80.9 \%)$ of the 2615 patients undergoing PCI enrolled in the TAMIS-II, 107 of whom had renal insufficiency. The baseline characteristics are shown in Table I. The patients who had renal insufficiency were older or there were more females compared to those who did not have renal insufficiency. The patients who had renal insufficiency were more frequently dependent in ADLs; they had lower BMI values and higher heart rate on admission, lower prevalences of hypercholesterolemia and peptic ulcer, greater prevalences of diabetes, angina, previous heart failure, previous renal failure, previous cerebrovascular disease, aortic aneurysm, and worse clinical course including bleeding, shock, Killip class $\geqq 3$, pulmonary edema, higher max CPK, and lower ejection fraction. There were fewer smokers among the patients who had renal insufficiency. In addition, the patients who had renal insufficiency frequently had a multivessel coronary disease.

Table II shows the procedural characteristics of the subjects. The use of a vasopressor, IABP, or mechanical ventilation was more likely in the patients with renal insufficiency, while thrombolytic use was less likely. 
Table I. Baseline Characteristics and Clinical Features of the Subjects

\begin{tabular}{|c|c|c|c|c|c|c|c|}
\hline & \multicolumn{3}{|c|}{ Male $(n=1675)$} & \multicolumn{3}{|c|}{ Female $(n=441)$} & \multirow{2}{*}{$\begin{array}{c}P \\
\mathrm{CC}<30 \\
\text { versus } \geqq 30\end{array}$} \\
\hline & $\begin{array}{l}\mathrm{CC}<30 \\
(n=63)\end{array}$ & $\begin{array}{c}\mathrm{CC} \geqq 30 \\
(n=1612)\end{array}$ & $P$ & $\begin{array}{l}\mathrm{CC}<30 \\
(n=44)\end{array}$ & $\begin{array}{l}\mathrm{CC} \geqq 30 \\
(n=397)\end{array}$ & $P$ & \\
\hline Age (years) & $70.98 \pm 11.66$ & $62.16 \pm 10.74$ & $<0.01$ & $74.82 \pm 11.27$ & $70.30 \pm 9.37$ & $<0.01$ & $<0.01$ \\
\hline Independent ADL (\%) & 84.13 & 97.15 & $<0.01$ & 90.91 & 95.21 & NS & $<0.01$ \\
\hline Body temperature $\left({ }^{\circ} \mathrm{C}\right)$ & $36.07 \pm 0.83$ & $36.25 \pm 0.70$ & NS & $36.62 \pm 4.76$ & $36.18 \pm 0.75$ & NS & NS \\
\hline Heart rate (beats/min) & $84.29 \pm 24.78$ & $78.49 \pm 17.44$ & $<0.05$ & $86.34 \pm 22.80$ & $78.87 \pm 17.74$ & $<0.05$ & $<0.01$ \\
\hline Systolic blood pressure (mmHg) & $124.54 \pm 33.80$ & $129.36 \pm 47.03$ & NS & $128.48 \pm 36.72$ & $132.41 \pm 28.56$ & NS & NS \\
\hline Body mass index $\left(\mathrm{kg} / \mathrm{m}^{2}\right)$ & $21.18 \pm 3.14$ & $23.93 \pm 3.42$ & $<0.01$ & $20.77 \pm 3.87$ & $22.95 \pm 3.55$ & $<0.01$ & $<0.01$ \\
\hline \multicolumn{8}{|l|}{ Medical history (\%) } \\
\hline Hypertension & 49.21 & 42.25 & NS & 54.55 & 49.12 & NS & NS \\
\hline Hypercholesterolemia & 6.35 & 19.23 & $<0.05$ & 9.09 & 22.67 & $<0.05$ & $<0.01$ \\
\hline Diabetes & 34.92 & 27.85 & NS & 43.18 & 25.94 & $<0.05$ & $<0.05$ \\
\hline Angina & 25.40 & 12.22 & $<0.01$ & 18.18 & 13.60 & NS & $<0.01$ \\
\hline Arrhythmia & 9.52 & 5.46 & NS & 2.27 & 4.28 & NS & NS \\
\hline Heart failure & 11.11 & 2.11 & $<0.01$ & 18.18 & 4.03 & $<0.01$ & $<0.01$ \\
\hline Myocardial infarction & 12.70 & 9.49 & NS & 9.09 & 6.30 & NS & NS \\
\hline Smoking & 41.27 & 66.63 & $<0.01$ & 20.45 & 21.41 & NS & $<0.01$ \\
\hline Cerebrovascular disease & 20.63 & 8.50 & $<0.01$ & 13.64 & 10.33 & NS & $<0.01$ \\
\hline COPD & 3.17 & 1.05 & NS & 0.00 & 0.50 & NS & NS \\
\hline Aortic aneurysm & 3.17 & 0.93 & NS & 2.27 & 0.50 & NS & $<0.05$ \\
\hline Peptic ulcer & 3.17 & 10.79 & NS & 2.27 & 4.79 & NS & $<0.05$ \\
\hline Cancer & 4.76 & 4.40 & NS & 4.55 & 5.29 & NS & NS \\
\hline Allergy & 6.35 & 6.20 & NS & 2.27 & 4.79 & NS & NS \\
\hline Dementia & 3.17 & 0.37 & $<0.01$ & 0.00 & 1.51 & NS & NS \\
\hline End-of-life stage & 0.00 & 0.12 & NS & 0.00 & 0.25 & NS & NS \\
\hline \multicolumn{8}{|l|}{ Clinical course $(\%)$} \\
\hline Bleeding & 25.40 & 16.07 & $<0.05$ & 22.73 & 18.64 & NS & $<0.05$ \\
\hline Shock & 46.03 & 17.06 & $<0.01$ & 31.82 & 15.37 & $<0.01$ & $<0.01$ \\
\hline Killip class $\geqq 3$ & 58.73 & 30.09 & $<0.01$ & 52.27 & 33.75 & $<0.05$ & $<0.01$ \\
\hline Pulmonary edema (x-ray) & 42.86 & 20.84 & $<0.01$ & 40.91 & 25.69 & $<0.05$ & $<0.01$ \\
\hline \multicolumn{8}{|l|}{ Locations of MI (UCG) (\%) } \\
\hline Antero/septal & 50.79 & 47.77 & NS & 40.91 & 48.11 & NS & NS \\
\hline Lateral & 9.52 & 7.20 & NS & 9.09 & 5.79 & NS & NS \\
\hline Posterior & 17.46 & 13.40 & NS & 9.09 & 9.82 & NS & NS \\
\hline Inferior & 36.51 & 36.85 & NS & 29.55 & 38.29 & NS & NS \\
\hline Subendocardial & 0.00 & 0.50 & NS & 0.00 & 1.51 & NS & NS \\
\hline Others & 6.35 & 2.79 & NS & 4.55 & 2.02 & NS & NS \\
\hline Max CPK (IU/L) & $4916.53 \pm 8266.73$ & $2879.13 \pm 2854.87$ & $<0.01$ & $2705.80 \pm 5206.40$ & $2300.34 \pm 2531.75$ & NS & $<0.01$ \\
\hline UCG-EF $(\%)$ & $45.61 \pm 15.08$ & $52.45 \pm 12.77$ & $<0.01$ & $50.44 \pm 12.12$ & $53.20 \pm 12.47$ & NS & $<0.01$ \\
\hline \multicolumn{8}{|c|}{$\begin{array}{l}\text { Number of coronary arteries narrowed } \\
>75 \% \text { in AHA classification } \\
\text { (Angiographic data) }(\%)\end{array}$} \\
\hline 1 & 31.75 & 62.03 & & 52.27 & 53.40 & & \\
\hline$>1$ & 63.49 & 33.62 & $<0.01$ & 47.73 & 42.82 & 0.4355 & $<0.01$ \\
\hline Left main & 3.17 & 1.67 & 0.3735 & 2.27 & 0.50 & 0.1773 & NS \\
\hline
\end{tabular}

Data are presented as the mean value \pm SD or percentage of subjects.

$\mathrm{CC}$ indicates creatinine clearance; COPD, chronic obstructive pulmonary disease; MI, myocardial infarction; ADL, activity of daily living;

UCG, ultrasound-echocardiogram; EF, ejection fraction; and AHA, American Heart Association.

The unadjusted and multivariable-adjusted results of in-hospital mortality are shown in Table III. The patients who had renal insufficiency had roughly 15 times the in-hospital mortality rate of those without renal insufficiency. According to the multivariate analysis, renal insufficiency was identified as an independent predictor of in-hospital death. 
Table II. Procedural Characteristics of the Subjects

\begin{tabular}{lccccccc}
\hline & \multicolumn{3}{c}{ Male $(n=1675)$} & \multicolumn{3}{c}{ Female $(n=441)$} & \\
\cline { 2 - 6 } & $\begin{array}{l}\mathrm{CC}<30 \\
(n=63)\end{array}$ & $\begin{array}{c}\mathrm{CC} \geqq 30 \\
(n=1612)\end{array}$ & $P$ & $\begin{array}{c}\mathrm{CC}<30 \\
(n=44)\end{array}$ & $\begin{array}{c}\mathrm{CC} \geqq 30 \\
(n=397)\end{array}$ & $P$ & CC $<0$ versus $\geqq 30$ \\
& 93.65 & 89.95 & $\mathrm{NS}$ & 90.91 & 86.40 & $\mathrm{NS}$ & $\mathrm{NS}$ \\
Transfer to ICU/CCU & 1.59 & 15.76 & $<0.01$ & 6.82 & 8.31 & $\mathrm{NS}$ & $<0.01$ \\
Thrombolytics & 68.25 & 27.48 & $<0.01$ & 50.00 & 29.72 & $<0.05$ & $<0.01$ \\
Vasopressor & 34.92 & 16.69 & $<0.01$ & 27.27 & 17.13 & $\mathrm{NS}$ & $<0.01$ \\
IABP & 39.68 & 7.44 & $<0.01$ & 22.73 & 7.30 & $<0.01$ & $<0.01$ \\
Mechanical ventilation & 87.30 & 90.14 & $\mathrm{NS}$ & 86.36 & 88.16 & $\mathrm{NS}$ & $\mathrm{NS}$ \\
Acute PCI & 4.76 & 9.93 & $\mathrm{NS}$ & 6.82 & 9.57 & $\mathrm{NS}$ & $\mathrm{NS}$ \\
Stent placement & & & & & &
\end{tabular}

Data are presented as the percentage of subjects.

ICU/CCU indicates intensive care unit/coronary care unit; PCI, percutaneous coronary intervention; and IABP, intra-aortic balloon pump.

Table III. Crude and Multivariable-adjusted Odds of Death During Hospitalization

\begin{tabular}{|c|c|c|c|c|c|c|c|c|c|}
\hline \multicolumn{4}{|c|}{ No. of deaths } & \multirow[b]{2}{*}{$\begin{array}{l}\text { Odds ratio } \\
\text { unadjusted }\end{array}$} & \multirow[b]{2}{*}{$95 \% \mathrm{CI}$} & \multirow[b]{2}{*}{$\begin{array}{c}\text { Odds ratio } \\
\text { adjusted for age }\end{array}$} & \multirow[b]{2}{*}{$95 \% \mathrm{CI}$} & \multirow[b]{2}{*}{$\begin{array}{c}\text { Odds ratio adjusted } \\
\text { for age and } \\
\text { other variables* }\end{array}$} & \multirow[b]{2}{*}{$95 \% \mathrm{CI}$} \\
\hline $\begin{array}{l}\mathrm{CC}<30 \\
(n=107)\end{array}$ & $\%$ & $\begin{array}{c}\mathrm{CC} \geqq 30 \\
(n=2009)\end{array}$ & $\%$ & & & & & & \\
\hline 32 & 29.91 & 56 & 2.79 & 14.88 & $9.099-24.333$ & 11.227 & $6.673-18.886$ & 5.362 & $1.099-26.160$ \\
\hline
\end{tabular}

$\mathrm{CC}$ indicates creatinine clearance.

*Controlling for age, gender, activity of daily living, heart rate, body mass index, medical history, max CPK, ejection fraction, and angiographic data.

\section{DiscuSSION}

Limited data exist in Japan regarding the association between renal dysfunction and the risk of in-hospital death in AMI patients undergoing PCI. In this large-scale prospective study we examined the influence of renal insufficiency on in-hospital outcomes in Japanese patients with AMI who underwent PCI.

Our study identified an association between the greater frequency of worse clinical conditions, such as age, diabetes mellitus, and angina, with renal insufficiency. Possible explanations for this interaction include the fact that age and diabetes mellitus are the well-established leading cause of renal insufficiency ${ }^{2)}$ and the increased prevalence of coronary artery disease such as angina in patients with renal insufficiency is partly due to the increased presence of such risk factors of renal insufficiency. In our study, patients with renal insufficiency were more likely to be female, have a lower BMI, or be dependent in their ADLs, which may 
be due to a higher incidence of older age or a greater prevalence of chronic illness.

Also, patients with renal insufficiency showed worse clinical courses, such as a greater prevalence of bleeding, shock, or congestive heart failure on presentation. Although a greater prevalence of risk factors such as advanced age or diabetes mellitus or a greater number of diseased coronary vessels may contribute to the worse clinical courses, ${ }^{14,15)}$ it is also possible, as previously suggested, that advanced renal dysfunction independently contributes to increased risk of cardiovascular mortality, as well as adverse cardiac events ${ }^{4)}$ and bleeding. ${ }^{7)}$ It is also possible that the difference in pump failure may be due to unsuccessful PCI or a new adverse cardiac event such as restenosis or recurrent angina, ${ }^{1,4)}$ although this study did not assess the rate of procedural success such as Thrombolysis in Myocardial Infarction (TIMI) flow in the culprit coronary artery soon after coronary intervention. These offer a good explanation of our results.

Our results showed that vasopressors, IABP, and/or mechanical ventilation were prescribed more often in patients with renal insufficiency. The higher rates of such treatment may be due to the more frequent occurrence of severe heart failure among patients with renal insufficiency. Thrombolytics, on the other hand, were prescribed less in patients with renal insufficiency. Although the reasons for less thrombolytic use among patients with renal insufficiency are not well known ${ }^{8)}$ one possible explanation for the lower use is that they were avoided in patients with renal insufficiency due to the greater prevalence of contraindications such as older age, history of cerebrovascular disease, or present bleeding among those with renal insufficiency. ${ }^{8,16)}$

The present study demonstrates that in-hospital mortality after AMI in patients was higher among patients with renal insufficiency than among those without after adjustment for age and other predictors of mortality. Best, et $a l^{4)}$ investigated the relationship between renal insufficiency and clinical outcomes such as in-hospital mortality in 5327 patients undergoing PCI. In multivariable regression models, the investigators found that in-hospital mortality was significantly associated with renal insufficiency. The present study expands on the existing literature by demonstrating a relationship between renal insufficiency and inhospital mortality rate in a Japanese population with AMI. The principal finding of our study is that renal insufficiency significantly increases the risk of in-hospital death among Japanese AMI patients undergoing PCI. These findings suggest that the presence of baseline renal insufficiency should alert the clinician to a significantly increased risk for in-hospital death in Japanese AMI patients undergoing PCI, which in turn may support more intensive medical management.

The potential limitations of this study should be considered in the interpretation of these results. First, because the present study was conducted in a limited 
area or different country compared to other studies, further studies are needed to understand treatment allocation in AMI patients with renal insufficiency in Japan and to determine the extent of the differences in the treatment allocation between Japan and Western countries. Second, the present study is limited by inadequate accounting for other potential predictors of hospital outcomes because we did not measure several potentially important indicators of the severity of the AMI, such as infarct size or the degree of ST segment elevation. Lastly, the omission of detailed clinical assessments of renal function limits our study results, because we were unable to determine whether the patients had acute renal dysfunction or chronic kidney disease, or are on dialysis.

Conclusion: We conducted a prospective study to investigate the difference in inhospital mortality in patients with and without renal insufficiency undergoing PCI in Japan. We found that the in-hospital mortality rate was significantly higher in patients with renal insufficiency than in those without using multivariate analysis. Further studies are needed to identify the difference.

\section{ACKNOWLEDGMENT}

The authors thank Ms. Noriko Sano for her assistance with analyzing the data.

\section{REFERENCES}

1. Koyanagi T, Nishida H, Kitamura M, et al. Comparison of clinical outcomes of coronary artery bypass grafting and percutaneous transluminal coronary angioplasty in renal dialysis patients. Ann Thorac Surg 1996; 61: 1793-6.

2. Centers for Disease Control and Prevention (CDC). Incidence of end-stage renal disease among persons with diabetes--United States, 1990-2002. MMWR Morb Mortal Wkly Rep 2005; 54: 1097-100.

3. Wolfe RA, Port FK, Webb RL, et al. Excerpts from the United States Renal Data System 1998 annual data report: II. Incidence and prevalence of ESRD. Am J Kidney Dis 1998; 32: S38-49.

4. Best PJ, Lennon R, Ting HH, et al. The impact of renal insufficiency on clinical outcomes in patients undergoing percutaneous coronary interventions. J Am Coll Cardiol 2002; 39: 1113-9.

5. Kahn JK, Rutherford BD, McConahay DR, Johnson WL, Giorgi LV, Hartzler GO. Short- and long-term outcome of percutaneous transluminal coronary angioplasty in chronic dialysis patients. Am Heart J 1990; 119: 484-9.

6. Collins AJ, Li S, Ma JZ, Herzog C. Cardiovascular disease in end-stage renal disease patients. Am J Kidney Dis 2001; 38: S26-9. (Review)

7. Sadeghi HM, Stone GW, Grines CL, et al. Impact of renal insufficiency in patients undergoing primary angioplasty for acute myocardial infarction. Circulation 2003; 108: 2769-75.

8. Masoudi FA, Plomondon ME, Magid DJ, Sales A, Rumsfeld JS. Renal insufficiency and mortality from acute coronary syndromes. Am Heart J 2004; 147: 623-9.

9. Wilson S, Foo K, Cunningham J, et al. Renal function and risk stratification in acute coronary syndromes. Am J Cardiol 2003; 91: 1051-4.

10. Shlipak MG, Heidenreich PA, Noguchi H, Chertow GM, Browner WS, McClellan MB. Association of renal insufficiency with treatment and outcomes after myocardial infarction in elderly patients. Ann Intern Med 2002; 137: 555-62. 
11. Herzog CA, Ma JZ, Collins AJ. Poor long-term survival after acute myocardial infarction among patients on long-term dialysis. N Engl J Med 1998; 339: 799-805.

12. Hirakawa Y, Masuda Y, Kuzuya M, Iguchi A, Kimata T, Uemura K. Age differences in the delivery of cardiac management to women versus men with acute myocardial infarction. Int Heart J 2006; 47: 209-17.

13. Cockroft DW, Gault MH. Prediction of creatinine clearance from serum creatinine. Nephron 1976; 16: 31-41.

14. Woon VC, Lim KH. Acute myocardial infarction in the elderly- the differences compared with the young. Singapore Med J 2003; 44: 414-8.

15. Fujiwara K, Hiasa Y, Takahashi T, et al. Influence of diabetes mellitus on outcome in the era of primary stenting for acute myocardial infarction. Circ J 2002; 66: 800-4.

16. Ryan TJ, Anderson JL, Antman EM, et al. ACC/AHA guidelines for the management of patients with acute myocardial infarction. A report of the American College of Cardiology/American Heart Association Task Force on Practice Guidelines (Committee on Management of Acute Myocardial Infarction). J Am Coll Cardiol 1996; 28: 1328-428. 